\title{
Evaluación de la comprensión de los paneles interpretativos en parajes naturales
}

\author{
Comprehension assessment of informative panels on natural landscape
}

\author{
Yaiza Serna Sans (1), Jorge Morato LaRa (1), Sonia Sánchez Cuadrado (2) \\ (1) Universidad Carlos III de Madrid, España, jmorato@gmail.com; \\ (2) Sonia Sánchez-Cuadrado, Universidad Complutense de Madrid, España, sscuadrado@ucm.es
}

\begin{abstract}
Resumen
Se analiza la percepción de la comprensibilidad de los paneles informativos. Tras una revisión de la literatura se han extraído características relacionadas con la legibilidad tipográfica y la lecturabilidad, además de recomendaciones sobre el diseño. Se han recopilado cuarenta paneles de parajes naturales españoles y se han extraído automáticamente los valores de legibilidad. Por otro lado se han calculado manualmente las características recomendadas para estos paneles. Además se han encuestado a cinco personas por ruta, con el fin de conocer su percepción de la información suministrada. Por último un clasificador muestra que esta percepción depende tanto de la legibilidad como de la lecturabilidad. Se ha creado un modelo predictivo con un grado de éxito del $75 \%$. Se trata de un análisis realizado sobre una tipología escasamente estudiada, pero con impacto económico y cultural. El estudio puede ayudar a la mejora de la información aportada en estos soportes en diseños futuros.
\end{abstract}

Palabras clave: Paneles interpretativos. Diseño. Lecturabilidad. Legibilidad. Percepción de la comprensión. Turismo. Paisajes naturales.

\section{Introducción}

De acuerdo a la Ley 13/1985 de Patrimonio Histórico, en su Título Preliminar, una prioridad básica es la conservación y la difusión de los recursos. La Ley de Patrimonio Histórico incluye no solo inmuebles y objetos artísticos, sino también parajes naturales o zonas con valor histórico o cultural. El valor para el público de estos recursos depende del grado en que estos elementos son mostrados al usuario, y por tanto de cómo se comunique la información a los visitantes de los espacios culturales y naturales.

Un aspecto en absoluto desdeñable es su impacto en el turismo. El gasto por turistas internacionales que visitaron España en 2017 fue de 86.823 millones de euros, mientras que el de residentes fue de 44.233 millones (INE, 2018 a; 2018 b). Aunque en el "Motivo del Viaje" no se recoge el concepto "turismo de naturaleza" o similar, si se establece una aproximación mediante estudios en las comunidades autónomas. Así el

\begin{abstract}
A methodology to assess the comprehension of interpretation signs is proposed. A set of parameters to evaluate the legibility and readability characteristics collected from the literature are classified. Forty use items on panels from walking routes from Spain have been collected and tested with our methodology. Later, these parameters were computed semi-automatically, and the degree of compliance regarding these aspects was calculated. Also, a survey was performed to study the visitors' experience. Panels from each track have been evaluated by five people in three levels, under a set of established criteria. A classifying model has been computed showing that comprehension depends on both legibility and readability. Model accuracy is 0.75 . The results help us to establish the relative importance of each aspect. Interpretative panels have a cultural value with socio-economic impact. This study could be helpful to design better informative panels in the future.
\end{abstract}

Keywords: Heritage interpretation. Informative panels on sightseeing routes. Legibility. Readability. Comprehension. Tourist information. Natural landscapes.

Gobierno del Principado de Asturias (2017) indica que de los turistas que los visitan el $71,4 \%$ visita pueblos o lugares, y que concretamente el $19,2 \%$ realiza rutas cortas de senderismo. El gasto medio por turista es 548,6 euros por estancia. Un estudio similar en la Comunidad Valenciana (Generalitat Valenciana, 2015) estimó en siete millones las visitas para realizar senderismo, aunque no fuera este el motivo principal del viaje. De este modo, la Agencia Valenciana indica que el Ecoturismo es un producto emergente en el periodo 2010-2020. El Ministerio de Agricultura y Pesca, Alimentación y Medio Ambiente (2017) ha tratado de recopilar distintas fuentes sobre turismo de naturaleza y rural, a fin de determinar el impacto económico y social. Así se indica que se ha pasado de 2,7 millones de viajeros en 2009 a 3,6 en 2016. El gasto directo estimado para el turismo de naturaleza se estima en 9.000 millones, cifra incrementada por las actividades periféricas a este gasto, calculado en 8.600 millones adicionales. 
En relación al diseño de los paneles y el modo de comunicar a los visitantes información sobre el patrimonio, es importante el concepto de "interpretación". A partir de los años 50 cobra fuerza esta idea, que trata de potenciar que los recursos se muestren de forma que conecten de un modo efectivo con el público. En 1957, Freeman Tilden (1977) popularizó el concepto "Interpretación", que venía siendo utilizado anteriormente, para denotar las estrategias para conseguir este objetivo, sobre todo en lo relativo a elementos naturales. Tilden propuso un conjunto de principios que motivarán el interés del visitante que accede a un recurso natural. Así, el primer principio es que cualquier información no relacionada con el visitante será estéril, o el segundo, que señala que la información debe ser percibida por el visitante como una revelación (Tilden, 1977, p. 9):

I. Any interpretation that does not somehow relate what is being displayed or described to something within the personality or experience of the visitor will be sterile.

II. Information, as such, is not Interpretation. Interpretation is revelation based upon information. But they are entirely different things. However, all interpretation includes information.

Como se puede apreciar, el foco se centra en la percepción del visitante. Así, se define la interpretación como una actividad educativa que utiliza el contacto directo y medios ilustrativos, evitando un mero listado de hechos relativos al objeto (Tilden, 1977, p. 8):

An educational activity which aims to reveal meanings and relationships through the use of original objects, by firsthand experience, and by illustrative media, rather than simply to communicate factual information.

Con el texto interpretativo el propósito es inspirar y provocar al visitante para ampliar sus horizontes (Beck y Cable, 2011, p. 24): "The purpose of the interpretive story is to inspire and to provoke people to broaden their horizons."

En España el Ministerio de Agricultura y Pesca, a través del CENEAM, propone unas directrices para aplicar buenas prácticas en la Interpretación del Patrimonio (Ministerio de Medio Ambiente, 1999). La Asociación para la Interpretación del Patrimonio (2012) alude al $4^{\circ}$ principio de Tilden para enfatizar que se trata de provocar el interés del visitante. En concreto indica "la interpretación del patrimonio es el arte de revelar in situ el significado del legado natural y cultural al público que visita esos lugares en su tiempo libre". Por otro lado, Harpers Ferry Center (2009, p.15) indica que es el arte de provocar conexiones emocionales e intelectuales con los recursos mostrados. A este respecto Thomson y Harper (2000) indican que la interpretación facilita una conexión entre los intereses del visitante y la semántica del recurso. Moreira-Wachtel y Tréllez Solís (2013) indican varias estrategias para mejorar esta interacción, y enfatizan que los mensajes deben ser atractivos, breves, claros y directos. Un resumen de los factores que entran en juego se puede ver en Moscardo (1996).

El enfoque idóneo para provocar la curiosidad del visitante es la comunicación personal, aunque en la práctica no siempre resulta viable. Es en este contexto que los paneles interpretativos cobran especial relevancia, ya que con frecuencia son el único medio de comunicación (School of Tourism and Leisure Management, The University of Queensland, 2006). En el proceso de transmitir de forma efectiva información al visitante influyen múltiples factores, desde la inclinación del individuo hacia el tema, a sus experiencias personales (Ludwig, 2003).

Ludwig (2003, p. 22) recopiló un número considerable de criterios sobre el diseño de los paneles, entre los que destaca: la comprensión rápida, incluir elementos de interacción, provocar al visitante, relacionar lo que ve con su realidad habitual o sorprenderle para incentivar su motivación. Para ello recomienda textos cortos, impactantes y motivadores, no eludiendo las metáforas o comparaciones que relacionen lo que ve con su realidad cotidiana.

Las guías de recomendaciones sobre buenas prácticas no suelen estar sostenidas por evidencias científicas que avalen la importancia del criterio recomendado, aunque suelen reflejar el sentido común y la experiencia en el diseño. Un breve ejemplo puede ilustrar este punto: Bazán (2014) analiza siete publicaciones en los que cada uno propone un límite máximo de palabras recomendado para estas señales, el rango oscila desde un umbral de 70 hasta otro de 300 palabras. El mismo autor señala que el visitante tiende a no leer los paneles. El Scottish Natural Heritage, indica que las investigaciones (sin precisar) muestran que no se debe superar las 200 palabras. El Manual de Senderos Turísticos de Aragón recomienda hasta 300 palabras en paneles grandes y 150 en pequeños. Para el inglés, Coloquhoun (2005) sugiere que un lector medio lee 200 palabras en los 45 segundos que permanece de media leyendo, y 60 palabras si no es nativo. Como se puede observar los umbrales son variables y no parecen tener en cuenta ni la temática ni el lector.

Existen diferentes formas de medir la comprensión, desde tests de respuesta múltiple a preguntas que impliquen un proceso de inferencia (Kauchak et al, 2017). Siguiendo las directrices de 
Kauchak et al (2017) se distinguen dos dimensiones dificultad real y dificultad percibida. Esta segunda dimensión ya ha sido aplicada a paneles informativos de museos en Morato et al. (2018) donde los autores se centran en valorar la percepción del visitante de museos y de cómo de comprensible es el panel, en este sentido se valora la conexión del panel con el mundo del lector. En este estudio también nos hemos centrado en esta dimensión.

Como se ha indicado se va a evaluar la percepción del usuario sobre su comprensión del panel. Para ello hay que destacar dos dimensiones: legibilidad y lecturabilidad (Montesi, 2009). La primera es la capacidad de un texto de ser leído con facilidad, y depende básicamente de características tipográficas; la segunda hace referencia a la capacidad de ser comprendido. Obviamente la legibilidad tiene un rol en la comprensión que no puede omitirse. Es por esto que algunos autores prefieren hablar de legibilidad como la capacidad de ser comprendido, señalando dos subtipos: legibilidad tipográfica, para el aspecto visual; y la lingüística, para aspectos de la mejora de la comprensión mediante la redacción (Ferrando Belart, 2004). Esta última es la que denominamos más arriba lecturabilidad.

Adicionalmente hay otros aspectos que no serán tratados en este estudio. Por ejemplo la credibilidad del que habla es importante en la comunicación, pero se deben evitar palabras complejas o de duda (Ludwig, 2003). En Morato et al. (2018) se recopilan variables lingüísticas, como la profundidad del árbol sintáctico o la categoría morfológica, que afectan a la comprensión del texto.

En las siguientes secciones se explica el objetivo y método seguido. Se detallarán los indicadores utilizados y las encuestas para estimar la comprensión del texto. Se continúa con los resultados obtenidos, conclusiones alcanzadas y trabajos futuros.

\section{Desarrollo de la Investigación}

El presente estudio tiene como finalidad determinar el grado en el que los diferentes aspectos y variables que presenta un panel condicionan la percepción sobre el interés y comprensión del panel por parte del visitante. Para su consecución, se ha desarrollado en primer lugar un estudio exploratorio de la literatura científica y otros estudios sobre el diseño de paneles turísticos, con el propósito de que sirvan de referencia para seleccionar ítems de evaluación. A continuación se lleva a cabo un proceso de análisis y extracción de datos que permita evaluar los diferentes parámetros relacionados con la lecturabilidad y legibilidad en la literatura, y estudiar su correlación con el grado de aceptación que tienen para cada visitante. Por último, mediante técnicas de aprendizaje automático se crea un modelo que permite discernir en qué grado son críticos los parámetros presentes en las guías, según el modelo obtenido mediante la evaluación por los visitantes de los paneles.

Los pasos seguidos para el desarrollo de la investigación han sido:

1. Síntesis de criterios representados en la literatura: Se ha tomado como base el trabajo de Serna (2017), y se han incluido otros estudios de relevancia para el presente estudio.

2. Se han recopilado 40 paneles de 20 rutas distribuidas por seis Comunidades Autónomas (Castilla-León, Castilla-La Mancha, Aragón, Madrid, Andalucía, Navarra). La selección, tanto de rutas como de paneles, se ha realizado al azar.

3. Para el cómputo de palabras y frases se han digitalizado las imágenes y se han extraído los textos mediante un OCR. Debido al escaso contraste y condiciones lumínicas hubo que revisar este proceso manualmente.

4. Se han recopilado y estimado un total de 20 parámetros:

- Cálculo de la lecturabilidad: cálculo de Fernández-Huerta, número de frases, número de palabras, presencia de imágenes y mapas relevantes, y coherencia con otros carteles.

- Cálculo de la familiaridad: valor absoluto y porcentual de palabras de baja frecuencia.

- Se han analizado los siguientes criterios relativos a la legibilidad: tamaño de fuente adecuado; número de tamaños de fuente; número de tipos de fuente; utilización de Sans Serif; contraste de la fuente con el fondo; número de temas tratados en el panel; presencia del tema principal en el título; uso de cursiva; longitud del texto recomendada (Gobierno de Aragón, 2012); presencia de números; Interlineado suficiente; y consistencia en el uso de mayúsculas

5. Se ha desarrollado una encuesta a cinco personas por ruta, con seis preguntas para saber su percepción en la comprensión del texto.

6. Se ha aplicado un clasificador para analizar qué variables influyen en la percepción de comprensibilidad. De esta manera se tendrá una aproximación del potencial predictivo del resto de las variables. 


\subsection{Indicadores estudiados}

A continuación se muestran criterios utilizados en la literatura para estimar la lecturabilidad, familiaridad terminológica y legibilidad.

\subsubsection{Lecturabilidad}

Para estimar la lecturabilidad se suelen utilizar índices adaptados del inglés, una recopilación de los mismos se puede encontrar en RamírezPuerta et al. (2013). Estos tests se basan en el número de palabras por frase o de sílabas por palabra. Parten del supuesto de que los criterios que facilitan la comprensión a los lectores son las frases sencillas y breves, y las palabras con menos caracteres. En este trabajo hemos utilizado una de las fórmulas más utilizadas para el español, propuesta por Fernandez-Huerta (1959), y modificada por Law (2014).

$206.84-60$ * sílabas /palabras - 102 * frases/palabras

El valor devuelto por la fórmula se asocia a un valor de complejidad preestablecido, en concreto: $90-100$, muy fácil; 80-90, fácil; 70-80, algo fácil; 60-70, normal (para adulto); 50-60, algo difícil; 30-50, difícil; y 0-30, muy difícil.

Existen variaciones a estas fórmulas, como los índices de Fernández Huerta (FH), Szigriszt-Pazos (SP), Legibilidad $\mu$ (L $\mu$ ) o Inflesz (Morato et al, 2018). En la tabla I se muestra como todas ellas utilizan las mismas variables.

\begin{tabular}{|c|c|c|c|c|}
\hline Indicador & $\mathrm{FH}$ & $S P$ & $L \mu$ & Inflesz \\
\hline Total de palabras & & * & * & * \\
\hline $\mathrm{N}^{\circ}$ Palabras por oración & * & & & \\
\hline \multicolumn{5}{|l|}{ Palabras de 3 o más sílabas } \\
\hline Total oraciones & & * & & * \\
\hline \multicolumn{5}{|l|}{ Oraciones cada 100 palabras } \\
\hline Total sílabas & & * & & \\
\hline Sílabas por palabra & * & & & \\
\hline Media caracteres por palabra & & & * & \\
\hline \multicolumn{5}{|l|}{ Caracteres por palabra } \\
\hline \multicolumn{5}{|l|}{$\begin{array}{l}\text { Media caracteres cada } 100 \\
\text { palabras }\end{array}$} \\
\hline Varianza caracteres palabra & & & * & \\
\hline
\end{tabular}

Tabla I. Indicadores de dificultad de lectura para el español (Morato et al., 2018)

En general, estas fórmulas suelen emplear los elementos mostrados anteriormente: caracteres de la palabra, número de palabras, número de frases o número de frases. En la tabla IV se muestran, para cada cartel, el número de palabras, frases y valor para el índice de FernándezHuerta.

Asociado a estas métricas está la propuesta de Ludwig (2003). Este autor indica que el número de letras dividido entre el número de palabras debe ser menor que seis, aunque no indica literatura que lo avale, precisando que este valor lo haría comprensible por un escolar de doce años.

\subsubsection{Familiaridad del término}

Junto a estos criterios, un indicador de importancia creciente es la familiaridad (Marks et al, 2014). Es decir, cual es la frecuencia de uso de las palabras que componen el cartel. La lógica subyacente es que palabas infrecuentes son más difíciles de comprender que las usuales. Dicho de otro modo, mide como de frecuente es el uso de un termino entre los hablantes, para determinar el grado en que ese termino es comprendido (Newbold et al, 2010; Leroy y Endicott, 2012).

Para estimar la familiaridad del término en español se pueden utilizar distintos corpus (Morato et al, 2018): Freeling (Padro et al, 2010), CREA de la Real Academia Española o el listado de frecuencias basado en OpenSubtitles. Freeling es en realidad un analizador lingüístico que precisa de frecuencias terminológicas y lingüísticas para estimar la probabilidad de una categoría o función gramatical. El corpus CREA se ha realizado con ciento sesenta millones de formas, con textos escritos y orales producidos desde el año 1975. La procedencia es variada, desde transcripciones de audio procedente de radio y televisión, periódicos, libros, etc. Por último, Opensubtitles, son el resultado de la compilación de subtítulos de películas (Invoke, 2018).

Ante la ausencia de un umbral preestablecido que determine la infrecuencia de un término, se establece a efectos prácticos el mismo valor que en el estudio de Morato et al. (2018). La Tabla IV muestra la familiaridad del término en relación al corpus CREA de la RAE, basado en un umbral de 10 ocurrencias obteniéndose una ratio sobre el total de palabras del cartel.

\subsubsection{Legibilidad}

Se trata de criterios visuales y tipográficos que pueden dificultar la lectura del texto, detallados en la Tabla II. Criterios de Legibilidad. Para la recopilación de estas tipografías se han consultado diferentes guías y recomendaciones sobre cómo diseñar la información de manera idónea. No existen unas directrices unificadas por lo que para este estudio se ha extraído los criterios coincidentes como (ver tabla II). 


\begin{tabular}{|c|c|c|c|}
\hline Indicador & Valores & Recomendado & Referencia Criterio \\
\hline $\begin{array}{l}\text { Coherencia } \\
\text { conceptual } \\
\text { en la ruta }\end{array}$ & Si/no & $\begin{array}{l}\text { Se puede seguir el hilo } \\
\text { argumental }\end{array}$ & Serna Sans, 2017; Smithsonian, 2011 \\
\hline $\begin{array}{l}\text { Consistencia } \\
\text { en el uso } \\
\text { de mayúsculas }\end{array}$ & Si/no & Uso consistente & $\begin{array}{l}\text { Coates y Ellison, 2014; Ferrer et al., 2011; Kasperek, 2014; Serna } \\
\text { Sans, 2017; Smithsonian, } 2011\end{array}$ \\
\hline $\begin{array}{l}\text { Utilización } \\
\text { de Sans-Serif, } \\
\text { Arial, Helvética }\end{array}$ & $\mathrm{Si} / \mathrm{no}$ & $\begin{array}{l}\text { Tipografías sencillas, sin } \\
\text { adornos ni engrosamientos }\end{array}$ & $\begin{array}{l}\text { Coates y Ellison, 2014; Department of Justice, 2010; Diputación } \\
\text { Provincial de Huesca, 2009; Ludwig, 2003; Serna Sans, 2017; } \\
\text { Smithsonian, } 2011\end{array}$ \\
\hline $\begin{array}{l}\text { Presencia } \\
\text { de números }\end{array}$ & Si/no & $\begin{array}{l}\text { Se distinguen claramente del } \\
\text { resto de texto }\end{array}$ & $\begin{array}{l}\text { Centro de Recuperación de Personas con Discapacidad Física, } \\
\text { 2010; Serna Sans, 2017; 2010; Smithsonian, } 2011\end{array}$ \\
\hline Uso de cursiva & Si/no & Evitar su uso & $\begin{array}{l}\text { Coloquhoun, 2005; Kasperek, 2014; Serna Sans, 2017; } \\
\text { Smithsonian, 2011; Te Papa National Services Te Paerangi, } 2006\end{array}$ \\
\hline $\begin{array}{l}\text { Contraste } \\
\text { fuente fondo }\end{array}$ & $\begin{array}{l}\text { Alto/medio } \\
\text { /bajo }\end{array}$ & $\begin{array}{l}\text { Alto contraste. Evitar elementos } \\
\text { decorativos y cajas de color }\end{array}$ & $\begin{array}{l}\text { Coates y Ellison, 2014; Ferrer et al., 2011; School of Tourism and } \\
\text { Leisure Management. The University of Queensland, 2006; Serna } \\
\text { Sans, 2017; Smithsonian, } 2011\end{array}$ \\
\hline $\begin{array}{l}\text { Tamaños } \\
\text { de fuente }\end{array}$ & Numérico & $\begin{array}{l}\text { Diversas, frecuentemente tres } \\
\text { tamaños o menos }\end{array}$ & Bazán, 2014; Coates y Ellison, 2014; Serna Sans, 2017 \\
\hline $\begin{array}{l}\text { Mapas } \\
\text { aclaratorios }\end{array}$ & Si/no & $\begin{array}{l}\text { Informar de la orientación junto a } \\
\text { un elemento observable por el } \\
\text { visitante }\end{array}$ & $\begin{array}{l}\text { Harpers Ferry Center, 2009; School of Tourism and Leisure } \\
\text { Management. The University of Queensland, 2006; Serna, } 2017\end{array}$ \\
\hline $\begin{array}{l}\text { Tamaño } \\
\text { de fuente } \\
\text { adecuado }\end{array}$ & Si/no & $\begin{array}{l}\text { Diversas, frecuente con la } \\
\text { distancia al cartel (p. e. legible a } \\
\text { un metro de distancia) }\end{array}$ & $\begin{array}{l}\text { Campos López, 2016; Coloquhoun, 2005; Department of Justice, } \\
\text { 2010; Diputación Provincial de Huesca, 2009; Kasperek, 2014; } \\
\text { Ludwig, 2003; Serna Sans, 2017; Smithsonian, } 2011\end{array}$ \\
\hline $\begin{array}{l}\text { Número de } \\
\text { tipos de fuente }\end{array}$ & Numérico & $\begin{array}{l}\text { Diversas, recomendado dos } \\
\text { tipos o menos }\end{array}$ & Harpers Ferry Center, 2009; Kasperek, 2014; Serna Sans, 2017 \\
\hline $\begin{array}{l}\text { Interlineado } \\
\text { suficiente }\end{array}$ & Si/no & Espacio entre bloques y líneas & $\begin{array}{l}\text { Coates y Ellison, 2014; Coloquhoun, 2005; Department of Justice. } \\
\text { 2010; Harpers Ferry Center, 2009; Serna Sans, 2017; Smithsonian, } \\
\text { 2011; Te Papa National Services Te Paerangi, } 2006\end{array}$ \\
\hline $\begin{array}{l}\text { Temas } \\
\text { tratados en el } \\
\text { panel }\end{array}$ & Numérico & $\begin{array}{l}\text { Diversas, más de cinco temas no } \\
\text { suele ser recomendable }\end{array}$ & Bazán, 2014; Morales Miranda, 2008; Serna Sans, 2017 \\
\hline $\begin{array}{l}\text { Presencia } \\
\text { del tema } \\
\text { principal en el } \\
\text { título }\end{array}$ & Si/no & $\begin{array}{l}\text { Título informativo o motivador y } \\
\text { con una tipografía adecuada }\end{array}$ & $\begin{array}{l}\text { Bazan, 2014; Morales Miranda, 2008; Department of Conservation. } \\
\text { Te Papa Athawai, 2005; School of Tourism and Leisure } \\
\text { Management. The University of Queensland, 2006; Serna Sans, } \\
\text { 2017; Viguera, } 2013\end{array}$ \\
\hline $\begin{array}{l}\text { Imágenes } \\
\text { aclaratorias }\end{array}$ & Si/no & $\begin{array}{l}\text { Ayudan a la comprensión de la } \\
\text { información textual }\end{array}$ & $\begin{array}{l}\text { Ferrer et al., 2011; Paths for all, 2002; Tourism Nova Scotia, 2008; } \\
\text { School of Tourism and Leisure Management. The University of } \\
\text { Queensland, 2006; Serna, } 2017\end{array}$ \\
\hline $\begin{array}{l}\text { Longitud } \\
\text { del texto } \\
\text { adecuada }\end{array}$ & Si/no & $\begin{array}{l}\text { Muy diversas, en este trabajo se } \\
\text { ha optado por la directriz del } \\
\text { Gobierno de Aragón: } 300 \\
\text { palabras en paneles grandes y } \\
150 \text { en pequeños }\end{array}$ & $\begin{array}{l}\text { Alonso Fernández y García Fernández, 2010; Bazán, 2014; } \\
\text { Department of Conservation. Te Papa Athawai, 2005; Gobierno de } \\
\text { Aragón, 2012; Ludwig, 2003; Morales Miranda, 2008; Paths for all, } \\
\text { 2002; School of Tourism and Leisure Management. The University } \\
\text { of Queensland, 2006; Tourism Nova Scotia, 2008, Te Papa National } \\
\text { Services Te Paerangi, 2006; Serna Sans, 2017; Smithsonian, } 2011\end{array}$ \\
\hline
\end{tabular}

Tabla II. Criterios de Legibilidad considerados en el estudio

\begin{tabular}{ll}
\hline Consulta & Valores \\
\hline ¿Se comprende el texto fácilmente? & Alto: los valores del objeto expositivo son mostrados de forma comprensible \\
& Medio: el tema principal del objeto resulta comprensible, pero hay subtemas o vocabulario \\
& confuso \\
& Bajo: el tema principal no queda explicado de forma clara \\
\hline ¿Se lee el texto fácilmente? & Alto: se lee sin dificultad, la estructura y gráficos ayudan y motivan al lector \\
& Medio: se puede leer con algún esfuerzo \\
& Bajo: la organización de bloques es confusa, cuesta leer por tipografía y contraste \\
\hline ¿La longitud del texto & Alto: la longitud del texto es adecuada para explicar el elemento \\
es la adecuada? & Medio: la longitud es adecuada, pero de forma demasiado sucinta \\
& Bajo: la longitud del texto no se adecua a lo necesario para explicar el elemento \\
\hline ¿Se suministra información & Si: con la información facilita se alcanza a ver el interés del elemento \\
suficiente & Normal: se aprecia de forma general su importancia, pero no porque destaca sobre otros \\
para ver el alcance de lo & elementos \\
observado? & No: no queda clara la importancia para destacar el elemento o faltan elementos por explicar \\
\hline ¿Imágenes aclaratorias? & Si: tipología legible y significativa para comprender el texto \\
(Bazán, 2014) & No: no mejora la comprensión del tema o es redundante \\
\hline ¿Mapas aclaratorios? & Sí: tipología legible y significativa para comprender la ruta \\
Coloquhoun (2005) & No: no mejora la comprensión del tema \\
\hline
\end{tabular}

Tabla III. Criterios para asignar valores en la encuesta 


\begin{tabular}{|c|c|c|c|c|c|c|}
\hline Provincia & Título del panel & $\begin{array}{l}\text { Fernández } \\
\text {-Huerta }\end{array}$ & $\begin{array}{l}N^{\circ} \\
\text { frases }\end{array}$ & $\begin{array}{c}N^{\circ} \\
\text { palabras }\end{array}$ & $\begin{array}{c}\% \\
\text { Familiar }\end{array}$ & $\begin{array}{l}\text { Opinión } \\
\text { usuarios }\end{array}$ \\
\hline \multirow[t]{3}{*}{ Albacete } & De Cotillas a Cueva Ahumada & 46,08 & 10 & 336 & 7 & Alta \\
\hline & De Riópar al nacimiento del río Mundo & 59,09 & 10 & 241 & 7 & Media \\
\hline & Cicular Arguellite, Los Prados, Rincón Cabero & 53,8 & 15 & 396 & 7 & Media \\
\hline Ávila & Senda del Puerto del Peón & 74,09 & 44 & 589 & 5 & Alta \\
\hline $\begin{array}{l}\text { Burgos/S } \\
\text { oria }\end{array}$ & Senda del Río & 59,66 & 13 & 332 & 7 & Media \\
\hline Cádiz & Fuente de las Piletas & 71,45 & 15 & 185 & 5 & Alta \\
\hline \multirow[t]{3}{*}{ Córdoba } & Fuente del Abad & 70,55 & 43 & 366 & 5 & Media \\
\hline & Mirador del Toro en la Cuesta del Espino & 66,98 & 35 & 551 & 7 & Alta \\
\hline & Ruta de los Lagares de Moriles & 71,54 & 20 & 315 & 10 & Media \\
\hline \multirow[t]{4}{*}{ Cuenca } & Sendero de Palomera & 66,14 & 45 & 663 & 10 & Alta \\
\hline & Los Lagunillos & 47,29 & 23 & 366 & 9 & Baja \\
\hline & El Rodenal de Boniches & 62,86 & 23 & 409 & 7 & Alta \\
\hline & El Escalerón y la Raya & 71,19 & 31 & 265 & 11 & Alta \\
\hline Granada & Sierra de Huétor. Sendero Cerro de la Cruz & 74,26 & 40 & 313 & 4 & Alta \\
\hline \multirow[t]{15}{*}{ Huesca } & San Juan de la Peña y Monte Oroel & 60,14 & 4 & 75 & 8 & Baja \\
\hline & Ruta de Chimiachas. Parque de la Sierrra y Cañones del Guara & 58,08 & 25 & 305 & 6 & Alta \\
\hline & Sendero Accesible del Río Vero. Sierra y Cañones del Guara & 51,72 & 8 & 151 & 4 & Alta \\
\hline & Vida en el Carrizo II. Rutas del Molino & 68,36 & 17 & 199 & 2 & Alta \\
\hline & Vida en el Carrizo II. Rutas del Molino. Hogar dulce hogar & 70,37 & 14 & 104 & 6 & Alta \\
\hline & Vida en el Carrizo II. Rutas del Molino. Vida secreta, pero muy secreta & 64,84 & 27 & 291 & 9 & Alta \\
\hline & $\begin{array}{l}\text { Vida en el Carrizo II. Rutas del Molino. La enorme importancia de una } \\
\text { estrecha franja verde }\end{array}$ & 69,19 & 14 & 189 & 4 & Alta \\
\hline & Ruta III: oficios y tradiciones. El Grado & 59,33 & 7 & 197 & 2 & Alta \\
\hline & Ruta III: oficios y tradiciones. El Grado. El Barbero (Huesca) & 57,28 & 9 & 178 & 4 & Media \\
\hline & Ruta III: oficios y tradiciones. El Grado. Fases de la Luna & 77,8 & 18 & 202 & 1 & Media \\
\hline & El arte rupestre: abrigo de Arpán. Parque Cultural del río Vero & 54,41 & 24 & 486 & 6 & Alta \\
\hline & El arte rupestre y sus representaciones. Parque Cultural del río Vero & 59,35 & 32 & 443 & 6 & Media \\
\hline & Abrigo de Arpán. Arte Levantino. Parque Cultural del Río Vero & 59,07 & 22 & 315 & 6 & Media \\
\hline & Ruta al Tozal de Mallata. Parque Cultural del río Vero & 62,06 & 32 & 491 & 9 & Alta \\
\hline & Abrigos del Tozal de Mallata. Parque Regional del rio Vero & 62,24 & 16 & 260 & 8 & Alta \\
\hline \multirow[t]{3}{*}{ Jaén } & Ruta Cicloturísta de Bercho. Sendero del Hoyo de la Sierra & 70,9 & 28 & 368 & 8 & Media \\
\hline & Pegalajar. Geología & 57,56 & 7 & 225 & 7 & Media \\
\hline & Pegalajar. Chozo de la Serrezuela & 61,01 & 10 & 188 & 8 & Alta \\
\hline \multirow[t]{5}{*}{ Madrid } & Zarzamora. Virgen del Retamar & 60,43 & 10 & 136 & 7 & Alta \\
\hline & Fresno. Virgen del Retamar & 64,6 & 11 & 184 & 9 & Alta \\
\hline & Fauna y ecosistema del río. Virgen del Retamar & 53,39 & 8 & 184 & 7 & Alta \\
\hline & Área recreativa Virgen del Retamar & 61,87 & 14 & 182 & 4 & Alta \\
\hline & Villanueva del Pardillo al Puente del Retamar & 65,6 & 16 & 264 & 8 & Alta \\
\hline Navarra & Huarte-Araquil & 71,67 & 28 & 363 & 8 & Media \\
\hline Segovia & Puente de la Cantina: Camino de pesquerías & 66,84 & 6 & 162 & 4 & Alta \\
\hline Toledo & Desfiladero del Pedroso & 60,99 & 34 & 537 & 9 & Baja \\
\hline
\end{tabular}

Tabla IV. Medidas de lecturabilidad, familiaridad terminológica y percepción de la comprensión por los usuarios

- Coherencia conceptual en la ruta. Se puede seguir el hilo argumental. Las referencias son generales, en la mayoría de guías la recomendación es implícita, ya que destacan que todo debe tener relación (Smithsonian, 2011).

- Alternar el uso de mayúsculas, (Coates y Ellison, 2014; Ferrer et al., 2011; Kasperek, 2014; Smithsonian, 2011).

- Usar tipografía estándar Sans Serif (Coates y Ellison, 2014; Diputación Provincial de Huesca, 2009) o similares, como Helvética (Ludwig, 2018).

- Evitar tipografías demasiado adornadas o con elementos inusuales (Department of Justice,
2010) o tipografías con caracteres similares (Smithsonian, 2011).

- Números en cifras, no en letra, y con tipografía clara (Centro de Recuperación de Personas con Discapacidad Física, 2010; Smithsonian, 2011).

- Evitar o reducir el uso de itálica, al ser éste un estilo no accesible para personas con dificultades visuales, limitando su uso en el texto y, siempre que sea posible, recurriendo a alternativas como el subrayado o las comillas (Kasperek, 2014; Te Papa National Services Te Paerangi, 2006; Smithsonian, 2011). 
- Mantener un alto contraste entre elementos y fondo (Coates y Ellison, 2014; Ferrer et al., 2011; School of Tourism and Leisure Management. The University of Queensland, 2006; Smithsonian, 2011)

- No utilizar más de 3 tamaños diferentes de fuente, ya que puede generar desorden y confusión (Bazan, 2014; Coates y Ellison, 2014)

- En los mapas, mostrar la ruta y los puntos de interés en los mapas. Informar de la orientación indicando dónde está el norte, o con el punto "usted está aquí" junto a un elemento observable por el visitante (Harpers Ferry Center, 2009; School of Tourism and Leisure Management. The University of Queensland, 2006).

Además de los indicados, existen otras pautas comunes, pero algo más heterogéneas en sus recomendaciones, como:

- Tamaño de la fuente: para que el texto pueda leerse a un metro de distancia. Entre 1,4 y 2,8 cm (Diputación Provincial de Huesca, 2009), entre $4,5 \mathrm{~mm}$ de altura y 24 puntos de formato (Smithsonian, 2011), alrededor de 0,6 cm (Campos López, 2016). Ludwig (2003) indica que este en el entorno de los 48 puntos.

- Número de tipografías diferentes: se recomienda una o, como máximo, dos fuentes (Harpers Ferry Center, 2009). Preferentemente, un único tipo Sans Serif y una fuente con valor decorativo para las ilustraciones (Kasperek, 2014).

- Interlineado: en relación a este factor se afirma que el texto debe tener un espaciado suficiente (Harpers Ferry Center, 2009). Existen afirmaciones más específicas, como que el interlineado debe ser al menos un $20 \%$ superior al tamaño de fuente y el espacio entre letras debe ser consistente y suficiente para evitar que las letras se superpongan, (Smithsonian, 2011), o lo indicado por Te Papa National Services Te Paerangi (2006) que afirma que el interlineado debe ser entre un $130 \%$ y un $150 \%$ superior al tamaño de la fuente.

- Evaluar si el panel presenta cinco o menos ideas principales (Bazan, 2014; Morales Miranda, 2008).

- En otras fuentes se proponen pautas que pueden dar lugar a resultados dispares, por ejemplo obras que aconsejan la presencia de un titulo descriptivo que muestre la idea principal (Viguera, 2013), y obras que apuestan por títulos interpretativos que capten la atención del visitante (Bazan, 2014; Morales Miranda, 2008; School of Tourism and Leisure Management. The University of Queensland, 2006).
Dado que generalmente "los visitantes sólo leen el título, las imágenes y leyendas" (Department of Conservation. Te Papa Athawai, 2005, p.78), en el presente trabajo se ha considerado importante que sean descriptivos y los usuarios encuentren en ellos la información principal.

- Respecto a las imágenes, se valora si ayudan a la comprensión de la información textual: incorporando un pie explicativo (Ferrer et al., 2011; Paths for all, 2002 y Tourism Nova Scotia, 2008), o si son suficientemente expresivas por sí mismas (School of Tourism and Leisure Management. The University of Queensland, 2006). En el presente trabajo se ha evaluado si la imagen ayuda a comprender la información.

- Para finalizar, la longitud del texto sobre la ruta es uno de los ítems más estudiados y a la vez con pautas más diversas. Por ejemplo el Manual de Senderos Turísticos de Aragón (Gobierno de Aragón, 2012) recomienda 200300 palabras para paneles grandes y $100-150$ para paneles de menor dimensión. Por su parte Bazan (2014) recomienda 90 palabras, y afirma que los textos deben ser generalmente cortos, con 10 a 15 palabras por oración. (Morales Miranda, 2008 y School of Tourism and Leisure Management. The University of Queensland, 2006) aconsejan no exceder las 80 palabras por panel, y (Alonso Fernández y García Fernández, 2010) dividir la información en párrafos cortos de 25 a 75 palabras. Respecto a aquellas guías cuyo objeto son textos en inglés se recomienda textos de un máximo de 200 palabras (Paths for all, 2002 y Tourism Nova Scotia, 2008), o de entre 200 a 225 palabras (Department of Conservation. Te Papa Athawai, 2005). Por su parte (Te Papa National Services Te Paerangi, 2006) afirma que los párrafos no deben pasar las 50 palabras coincide con (Smithsonian, 2011) que una longitud de frase no debe ser superior a 25 palabras, (preferiblemente 15). Ludwig (2003) da una explicación a los distintos cálculos al indicar que la velocidad de lectura media es de 200 palabras por minuto, pero que el $90 \%$ de los visitantes no emplea más de 3 segundos, por lo que al menos el título tiene que tener diez palabras como máximo, y el texto en si menos de 100 palabras.

\subsubsection{Percepción de la comprensión}

Para estimar la percepción de la comprensión se realizó una encuesta a usuarios, de una forma similar a la propuesta por Ludwig (2003). La principal consulta es si consideran que comprenden el panel. El resultado de esta consulta es la variable 
dependiente del modelo de clasificación. La tabla III muestra las preguntas y los criterios para asignar los valores en la encuesta.

\subsubsection{Variables que influyen en la percepción de comprensibilidad}

El aprendizaje automático permite desarrollar técnicas que posibiliten a las máquinas emular las decisiones humanas. Para ello es necesario dotar al programa de ejemplos que faciliten extraer un patrón que pueda ser posteriormente reproducido con otros datos. En el estudio se ha tratado de analizar si era posible determinar aquellos parámetros que inciden más en la percepción del usuario, de manera que se pueda estimar la importancia de estos parámetros en la elaboración de carteles en el futuro. Weka es un entorno de aprendizaje automático desarrollado por la Universidad de Waikato. Se trata de un software libre dotado de un gran número de algoritmos de análisis de datos y modelado predictivo basado en el aprendizaje a través de los datos (Witten, Frank y Hall, 2011). En este estudio se experimentó con diversos algoritmos de clasificación (J48, PART, Random Forest y Ramdom Tree) con evaluación cruzada. Es decir se hacen distintas iteraciones en que un porcentaje de la colección se utiliza para aprendizaje y el resto para probar el modelo. La utilización de estos algoritmos y no otros se debe a su buen rendimiento y sencilla interpretación de los resultados. La aplicación de modelos de aprendizaje a la comprensión de textos ha sido descrita anteriormente en la literatura (Larsson, 2006).

Tras múltiples iteraciones los atributos considerados para la obtención del modelo predictivo, son: Coeficiente fórmula Fernández-Huerta, número de frases, número de palabras, valor absoluto y porcentaje de palabras con baja frecuencia en CREA, contraste letra-fondo, presencia imágenes significativas, presencia mapas relevantes, presencia de letra con tamaño legible, número de tamaños de fuentes, número de tipos de fuente, consistencia en el uso de mayúsculas, interlineado suficiente, uso de Sans-Serif o similares, uso de cursiva, longitud del texto, presencia del tema principal en el título y número de temas tratados en el cartel. Parámetros como los números y coherencia daban peores resultados en los modelos. Los datos sobre la relevancia para la comprensión de mapas como imágenes se han obtenido a partir de las consultas realizadas a los visitantes.

\section{Resultados}

La figura 1 muestra los resultados sobre si el visitante percibe el panel como: legible, comprensible, con longitud adecuada y con información suficiente. Además se ha incorporado el resultado del análisis de los paneles con las métricas de Fernández-Huerta como valor de referencia. Si bien en este caso, y a efectos prácticos de comparación, se han agrupado los valores "algo fácil" y "fácil" como "fácil", y "algo difícil" y "difícil" como "difícil". En ningún caso se obtuvieron valores correspondientes a "muy fácil" o "muy difícil".

Como se aprecia en la Figura 1 la legibilidad ( ¿se lee sin dificultad el panel?) y la comprensibilidad (¿se comprende bien el contenido del panel?) muestran cierta correlación. Sin embargo, la fórmula de Fernández-Huerta no parece dar una imagen precisa del grado de dificultad, seguramente porque obvia elementos importantes, como la información gráfica.

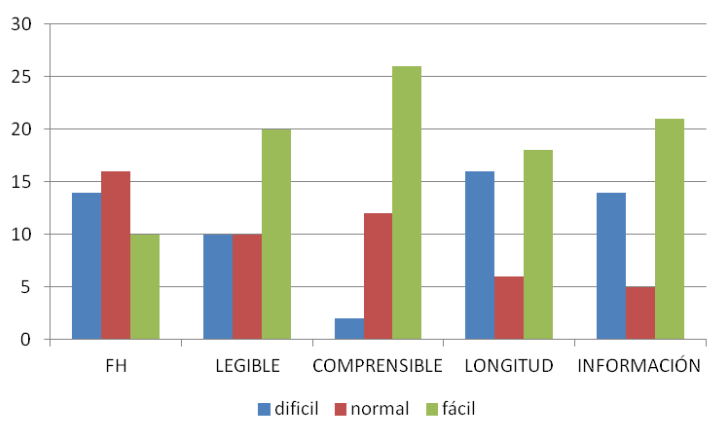

Figura 1. Lecturabilidad, familiaridad terminológica y opinión de los usuarios de los paneles

Para medir la tasa de acuerdo, el cálculo de la Kappa de Fleiss (1971) entre legibilidad y lecturabilidad ofrece un valor de $52,5 \%$, que de acuerdo a Landis y Koch (1977) es un acuerdo medio alto. La misma tasa de acuerdo se produce entre lecturabilidad e información suministrada. Sin embargo, si solo atendiéramos a la relación de lecturabilidad con longitud del texto, la tasa desciende a un 35\% (acuerdo medio).

En la tabla IV (ver apéndice) se muestran los resultados desglosados para los criterios de familiaridad del término y lecturabilidad. Además, se ha incluido la percepción de la comprensión por parte de los visitantes.

Tras realizar las distintas pruebas con Weka se seleccionó por la calidad de los resultados el algoritmo PART. Los mejores resultados se han obtenido para el siguiente conjunto de variables: coeficiente de Fernández-Huerta, número palabras con poca frecuencia en CREA, contraste, mapas relevantes, número de temas en el cartel, uso de Sans-Serif o similares, cumplimiento de pautas de longitud recomendada (según pautas Gobierno de Aragón (2012)), uso de cursivas y número de tamaños de fuente. El árbol de decisión obtenido indica que las variables con mayor peso son: uso de cursiva, longitud recomendada 
y número de temas en el cartel. Las instancias correctamente clasificadas han sido un $75 \%$, con una precisión del $71 \%$, una exhaustividad del $75 \%$ y una medida de la $\mathrm{F}$ del $73 \%$.

Los clasificadores utilizados, y entre ellos PART, facilitan conjuntos de reglas fácilmente legibles. A partir de los resultados obtenidos, estas reglas son extrapolables a recomendaciones para el diseño de carteles. Basándose en los resultados, las principales reglas para lograr un cartel que se perciba como más comprensible son: número de temas en el cartel (recomendado uno; rango en la muestra analizada: 1-8); uso de Sans-Serif o similar; frecuencia en Crea (recomendado $\leq 32$; rango en la muestra: 3-50); usar la longitud del texto recomendada; evitar cursivas; tamaños de Fuente (recomendado $\leq 3$; rango: 3-9); valor Fernández-Huerta (recomendado $\geq 61$; rango: 47,29$77,8)$; usar un contraste letra-fondo elevado, y utilizar mapas con información relevante

\section{Conclusiones}

La bibliografía para la redacción de paneles se centra en comprobar unas directrices, sin proveer fundamentación para las mismas. Por otra parte, las recomendaciones en torno al contenido informativo a incluir resultan generalistas y escasamente clarificadoras en su aplicación. En este estudio se ha señalado la importancia de algunas de estas directrices.

El cálculo de la Kappa de Fleiss sustenta la hipótesis de que la comprensión del texto está condicionada a que el visitante lo perciba como legible e informativo.

El criterio sobre la longitud del texto está presente en gran número de directrices, pero con disparidad de recomendaciones. Por otro lado, imágenes y mapas son elementos que permiten transmitir información adicional evitando un texto sobrecargado. En este trabajo se ha estudiado aspectos relacionados con la legibilidad y lecturabilidad. Se ha observado que aspectos presentes en las guías como contraste y uso de cursiva influyen en la comprensión. La longitud del texto también ha sido un parámetro significativo, pero otros como el número de temas tratados o la presencia de mapas significativos también han mostrado su relevancia, si bien no siempre están tratados en las guías.

Como trabajo futuro nos proponemos analizar el uso de las TIC en los paneles. Sin duda puede suponer una ayuda a la comprensión de los textos sin sobrecargar de información los paneles. Este estudio debería ser completado con una investigación sobre la disposición de los usuarios para utilizar elementos TIC con información adicional como códigos QR.

Los resultados muestran diferentes percepciones del cartel para distintos visitantes, en parte debidos a características socio-demográficas. Aunque estas raramente se abordan en los estudios que influyen en la comprensibilidad del texto. Una continuación del presente proyecto es incluir algunas de estas características.

\section{Agradecimientos}

El proyecto ha sido subvencionado por el Ministerio de Economía, Industria y Competitividad de España, con el número CSO2017-86747-R.

\section{Referencias}

Alonso Fernández, L.; García Fernández, I. (2010). Diseño de exposiciones: concepto, instalación y montaje. Madrid: Alianza.

Asociación para la Interpretación del Patrimonio. (2012). Asociación para la Interpretación del Patrimonio. http://www. interpretaciondelpatrimonio.com/

Bazán, H. G. (2014). La interpretación del patrimonio geomorfológico en los Picos de Europa: una propuesta para su aprovechamiento didáctico y geoturístico. Tesis doctoral, Universidad de Valladolid

Beck, L.; Cable, T. (2011). Interpretation for the 21st century: fifteen guiding principles for interpreting nature and culture. 3rd ed. Urbana (EEUU): Sagamore Pub.

Campos López, M. I. (2016). La interpretación del patrimonio como herramienta del turismo sustentable. (Tesis doctoral, Universidad de Quintana Roo). http://www.uqroo.mx/ cozumel/

Centro de Recuperación de Personas con Discapacidad Física. Albacete. (2010). Cómo elaborar textos de fácil lectura. http://www.crmfalbacete.es/crmfalbacete_01/index.htm

Coates, K.; Ellison, A. (2014). An Introduction to Information Design. Laurence King.

Coloquhoun, F. (2005). Interpretation Handbook and Standard: Distilling the essence. Department of Conservation. The Papa Atawhai, Wellington (NZ).

CREA. http://www.rae.es/recursos/banco-de-datos/crea

Department of Conservation. Te Papa Athawai (2005). Interpretation Handbook and Standard: Distilling the Essence. http://www.doc.govt.nz/

Department of Justice. (2010). ADA Standards for Accessible Design.https://www.ada.gov/regs2010/2010ADAStandar ds/2010ADAstandards.htm

Diputación Provincial de Huesca. (2009). Manual de Senderos Accesibles en la provincia de Huesca. https://issuu.com/rete21/docs/manual_senderos_accesibles_dph

España. Parlamento. (1985). Ley del Patrimonio Histórico Español (Ley 16/1985). Madrid: BOE.

Fernández Huerta, José (1959). Medidas sencillas de lecturabilidad. // Consigna, 214, 29-32.

Ferrando Belart, V. (2004). La legibilidad: un factor fundamental para comprender un texto. Atención Primaria, 34(3): 143-146.

Ferrer, M. de los Á.; Prieto, C. M.; Sánchez, J. I. (2011). ¿Una sociedad de la información en igualdad de condiciones? Evaluación al grado de inclusión social-digital que ofrecen las TIC desde la perspectiva de la usabilidad y la accesibilidad. // Quórum Académico. 8:16, 247-267. 
Fleiss, Joseph L. (1971). Measuring nominal scale agreement among many raters. // Psychological Bulletin. 76: 5, 378-382.

Generalitat Valenciana (2015). Turismo activo y de naturaleza en la Comunitat Valenciana. http://www.turisme.gva.es.

Gobierno de Aragón. (2012). Manual de Senderos Turísticos de Aragón. Aragón. http://www.boa.aragon.es

Gobierno del Principado de Asturias (5 sept. 2017). El gasto turístico se incrementa un $8 \%$ hasta alcanzar los 584,58 euros por persona y viaje. Nota de Prensa. http://www.asturias .es

Harpers Ferry Center. (2009). Wayside Exhibits. A guide to developing outdoor interpretive exhibits. https://www.nps. gov/index.htm

Harpers Ferry Center. (2017). Wayside Exhibit Planning. Harpers Ferry WV: National Park Service. https://www.nps. gov/hfc/products/waysides/way-process-planning.cfm

INE (2018a) Encuesta de Turismo de Residentes. http://www.ine.es

INE (2018b). Gasto de los turistas internacionales según motivo principal del viaje. http://www.ine.es

Invoke (2018) Frequency Word lists https://invokeit.wordpress.com/frequency-word-lists/

Kasperek, S. (2014). Sign Redesign: Applying Design Principles to Improve Signage in an Academic Library. II Pennsylvania Libraries. 2:1, 48-63. http://dx.doi.org/10. 5195/palrap.2014.54

Kauchak, David; Leroy, Gondy; Hogue, Alan (2017). Measuring text difficulty using parse-tree frequency. // Journal of the Association for Information Science and Technology. 68:9, 2088-2100.

Landis, J Richard; Koch, G Gary (1977). The measurement of observer agreement for categorical data. // Biometrics. 33:1, 159-74.

Larsson, Patrik (2006). Classification into Readability Levels Implementation and Evaluation. http://citeseerx.ist.psu.edu/vie wdoc/download;jsessionid=38303DD659563B139DEDDAD 11B4182BB?doi=10.1.1.102.5282\&rep=rep1\&type=pdf

Law, Gwillim (2011). Error in the Fernandez Huerta Readability Formula. https://linguistlist.org/issues/22/22-2332.html

Leroy, Gondy; Endicott, James E. (2012). Combining NLP with Evidence-based Methods to Find Text Metrics Related to Perceived and Actual Text Difficulty. // Proceedings of the 2Nd ACM SIGHIT International Health Informatics Symposium. 749-754. https://doi.org/10.1145/2110363. 2110452

Ley 16/1985, de 25 de junio, del Patrimonio Histórico Español. // BOE. 155 (29 de junio de 1985) 20342-20352

Ludwig, Thorsten (2003). Basic Interpretive Skills. The course Manual. http://www.videsgidi.Iv/faili/Basic_Interpretive_Skills_-_The_Course_Manual.pdf

Marks, C.B.; Doctorow, M.J.; Wittrock, M.C. (2014). Word Frequency and Reading Comprehension. // The Journal of Educational Research. 67:6, 259-262, DOI: 10.1080/0 0220671.1974.10884622

Ministerio Agricultura y Pesca, Alimentación y Medio Ambiente (2017). El turismo de naturaleza en España. Análisis y prospectiva. Serie Medio Ambiente, 9. http://www.mapama.gob.es

Ministerio de Medio Ambiente (1999). Libro Blanco de la educación ambiental en España en pocas palabras. http://www.mapama.gob.es/es/ceneam/recursos/documentos/pocas tcm30-77432.pdf

Montesi, M. (2009). Aproximación al documento textual desde la perspectiva de los estudios sobre el discurso. // Revista Española de Documentación Científica. 34:4, 92-105. doi: 10.3989/redc.2009.4.670
Morales Miranda, J. F. (2008). El sentido y metodología de la interpretación del patrimonio: La comunicación global del patrimonio cultural. 53-78. https://www.edu.xunta.gal

Morato, Jorge; Sánchez-Cuadrado, Sonia; Gimmelli, Paolo (2018). Estimación de la comprensibilidad en paneles de museos. // El profesional de la información. 27:3, 570-581.

Moreira-Wachtel, S., Tréllez Solís, E. (2013). La interpretación del patrimonio natural y cultural: Una visión intercultural y participativa. Lima: Ministerio del Ambiente Perú.

Moscardo, G. (1996). Mindful Visitors. Heritage and Tourism. // Annals of Tourism Research. 23:2, 376-397.

Newbold, N, McLaughlin, H, Gillam, L (2010). Rank by readability: Document weighting for information retrieval. // IRFC 2010, LNCS 6107. 20-30

Padró, Lluís; Collado, Miquel; Reese, Samuel; Lloberes, Marina; Castellón, Irene (2010). FreeLing 2.1: Five years of open-source language processing tools. // Procs. of the 7th Intl conf on language resources and evaluation (LREC'10). 931-936.

Paths for all. (2002). Producing Interpretive Panels. www.pathsforall.org.uk/

Ramírez-Puerta, M.R.; Fernández-Fernández, R.; Frías-Pareja, J.C.; Yuste-Ossorio, M.E.; Narbona-Galdó, S.; Penas-Maldonado, L. (2013). Análisis de legibilidad de consentimientos informados en cuidados intensivos. // Med Intensiva. 37:8, 503-509

Real Academia Española: Banco de datos (CREA) Corpus de referencia del español actual. http://www.rae.es

School of Tourism and Leisure Management. The University of Queensland. (2006). Interpretive Signage: Principles and Practice. https://signage.business.uq.edu.au/signage-interpretation.html

Scottish Natural Heritage: https://www.nature.scot/professional-advice/policy-guidance/natural-heritage-interpretation/producing-interpretive-panels

Serna-Sans, Y. (2017). Metodología para la evaluación de paneles en rutas y senderos turísticos: Ejemplo de aplicación. Tesis de Máster. Getafe: Universidad Carlos III.

Smithsonian. (2011). Smithsonian Guidelines for Accessible Exhibition Design. https://www.si.edu/accessibility

Te Papa National Services Te Paerangi. (2006). Writing Effective Interpretive Text. // Resources guides. 28, 1-20. https://www.tepapa.govt.nz/

Thomson, R, Harper, M (2000). Telling the Stories: Planning Effective Interpretive Programs for Properties Listed in the National Register of Historic Places bulletin. U.S. Department of the Interior, National Park System.https:// www.nps.gov/

Tilden, Freeman (1977) Interpreting Our Heritage. 3rd ed. Chapel Hill: University of North Carolina Press, Chapel Hill. https://is.muni.cz/

Tourism Nova Scotia. (2008). Tourism development. How-to guide Outdoor Interpretive Signage. Your guide to Connecting People \& Places. https://tourismns.ca/

Viguera, R. (2013). Estrategias y recursos de comunicación en la exposición permanente. // Sociedad: boletín de la Sociedad de Amigos de la Cultura de Vélez-Málaga. 12, 21-26. https://dialnet.unirioja.es/

Witten, lan H; Frank, Eibe; Hall, Mark A. (2011). Data Mining: Practical Machine Learning Tools and Techniques. 3rd ed. San Francisco (EE.UU.): Morgan Kaufmann Publishers.

Enviado: 2018-05-12. Segunda versión: 2018-07-23 Aceptado: 2018-09-05. 